\title{
RELACIÓN EMPÍRICA ENTRE LA TERAPIA RACIONAL EMOTIVO-CONDUCTUAL (TREC) DE ELLIS Y LA TERAPIA COGNITIVA (TC) DE BECK EN UNA MUESTRA COSTARRICENSE
}

\author{
Hannia Cabezas Pizarro \\ Catedrática de la Escuela de Orientación y Educación Especial de la Universidad de Costa Rica \\ San José, Costa Rica. \\ Leonor I. Lega \\ Catedrática de Psicología en el Department of Psychology, Saint Peter's College, Jersey City Campus, \\ USA.
}

Recibido 07-XI-2006

Resumen: En este estudio se explora una posible relación empírica entre la terapia racional emotivoconductual (TREC) de Ellis y la terapia cognitiva (TC) de Beck, dos de los modelos más conspicuos en el campo de la psicoterapia cognitiva actual.

Si bien este modelo utiliza el término "cognitivo" en forma tal que se encuentra asociado con el afecto, la fisiología y la conducta (Dobson, 1986), sostiene que las perturbaciones emocionales, en esencia, son causadas por el proceso cognitivo del individuo.

En esta investigación, se trabajó con una muestra de 200 estudiantes de la Universidad de Costa Rica de diferentes áreas a quienes se les aplicaron las escalas de: Attitudes and Beliefs Inventory (ABI) [Escala de Actitudes y Creencias], y el Beck Depression Inventory, $2^{a}$ edición (BDI-II) [Inventario de Depresión de Beck], escalas que fueron adaptadas al español.

El análisis estadístico de los datos confirma empíricamente la relación entre ambos modelos.

Palabras clave: Terapia racional emotivo-conductual, terapia cognitiva, relación empírica.
La terapia racional emotivo-conductual (TREC), se define como un sistema de terapia que ayuda a las personas a vivir mejor, a través del cual, puede minimizar sus problemas emocionales y sus conductas desadaptadas, lo que le permite autorrealizarse para tener una vida más plena y feliz. (Ellis y Bernard, 1990)

La filosofía descrita por Ellis (1955) fue publicada inicialmente bajo el nombre de terapia racional (Ellis, 1958), sin embargo, ha ido evolucionando a través de los años, convirtiéndose posteriormente en terapia racional emotiva (TRE), hasta nuestros días en que se le conoce con el nombre de terapia racional emotivo- conductual (TREC). (Ellis y Bernard, 1994)

Desde el año 1955 Albert Ellis conceptualizó los términos de ideas irracionales, creencias erróneas y poco asertivas acerca de uno mismo y las demás personas. Esto lo hizo a través de una conferencia presentada en el congreso anual de la American Psichological Association (APA) realizada en Chicago, Estados Unidos (Grieger y Boyd, 1980), siendo el creador de esta terapia. 


\begin{abstract}
The present study explores a potential empirical relationship between Ellis' rational-emotive behavior therapy (REBT) and Beck's cognitive therapy (CT), two of the most prominent models in the field of cognitive psychotherapy. Although this field uses the term "cognitive" in a way that is associated with affection, physiology, and behavior (Dobson, 1986), it maintains that the essence of emotional disturbance is caused by the cognitive process of an individual. This research study gave previously adapted Spanish versions of the Attitudes and Beliefs Inventory or $A B I$, and the Beck Depression Inventory- Second Edition (BDI-II) to a sample of 200 students from the University of Costa Rica.

Spearman's rho correlation coefficients were calculated, and their results were analyzed in terms of previously proposed theoretical relationships between the two models.
\end{abstract}

Key words: Rational-emotive behavior therapy, cognitive therapy, Attitudes and Beliefs Inventory, Beck Depression Inventory.
De acuerdo con Ellis y Abrahms (2001) la terapia racional emotiva, no sólo pretende, que sus teorías sean sujetas a comprobación, sino que enseña a las personas a

hacer lo mismo sobre sus teorías acerca de sí mismos y de los demás; a discutir sus propias ideas, a descubrir lo que lleva a resultados "buenos" o positivos, y a descartar aquellos que conducen a consecuencias “malas" o negativas. (Ellis y Abrahms, 2001, p. 30)

La TREC está basada en la idea de que tanto las emociones como las conductas de un individuo son producto de las creencias que posee y de la interpretación que haga de la realidad (Ellis, 1962). Por lo tanto, la meta fundamental de la TREC es la de asistir al paciente en la identificación de sus pensamientos "irracionales" o disfuncionales y ayudarle a que los sustituya por otros más "racionales" o eficientes, que le permitan lograr con más eficacia metas de tipo personal como el ser feliz, el poder establecer relaciones con otras personas, etc. (Ellis y Becker, 1982). La TREC examina además la filosofía básica del individuo, que constituye la base sobre la que surgen las inferencias sobre él mismo, sobre los demás y sobre el mundo en general.

El marco filosófico general de esta terapia se basa principalmente en la premisa estoica de que "la perturbación emocional no es creada por las situaciones sino por las interpretaciones de esas situaciones". (Epitectus, 1980)

El modelo terapéutico denominado $A B C$ se puede considerar, dentro de la TREC, como un marco de referencia muy amplio en donde se pueden conceptualizar los problemas psicológicos de los pacientes. El modelo ABC de la conducta fue descrito por Ellis en el año 1962. En este modelo se expone que hay un "acontecimiento activante" (A) que es interpretado por el individuo, quien desarrolla una serie de creencias ("Beliefs") (B) sobre él mismo. A partir de esas creencias se desarrollan las consecuencias $(\mathbf{C})$ que resultarían de la interpretación que el individuo hace de A. 
Las percepciones y concepciones erróneas, de lo que sucede realmente en el punto A, responden a patrones disfuncionales $\mathrm{y}$ dificultan el funcionamiento eficaz del individuo, por lo que no hay objetividad (B irracional), y ello causa en gran medida "consecuencias emocionales" (Ce) y/o "consecuencias conductuales" (Cc).

Desde el punto de vista cognoscitivo o filosófico, de acuerdo con Ellis y Abrahms (2001) se supone o se establece la hipótesis, que lo que concebimos como nuestras consecuencias o reacciones emocionales (C) se desencadenan principalmente de nuestras ideas o creencias conscientes o inconscientes $(\mathbf{B})$, de nuestras evaluaciones, y propias interpretaciones de lo que sucede en (A), y esto es lo que se ha denominado el modelo ABC de la conducta.

El pensamiento irracional (B) inicialmente se trataba como un concepto "global" pero con el tiempo ha ido evolucionando a ideas específicas. (Sutton-Simon, 1981)

Diferentes investigaciones llevadas a cabo en la década de 1970 (Ellis, 1973; 1975 ; 1976) identificaron once ideas irracionales las que posteriormente fueron agrupadas en tres inferencias: Tremendismo, Baja tolerancia a la frustración (BTF), y Condenación o "Evaluación global de la valía del ser humano" (Ellis, 1984; 1985). $\mathrm{El}$ "tremendismo" es la tendencia a resaltar excesivamente la parte negativa de un acontecimiento, es una conclusión exagerada y mágica que proviene de la creencia "esto no debería ser tan malo como es"; la "baja tolerancia a la frustración" es la tendencia a exagerar lo insoportable de una situación y a considerarla como insufrible, puesto que al presentarse elimina toda posibilidad ya sea presente y/o futura de ser feliz; la "condenación" es la tendencia a evaluar como "mala" la esencia humana, la valía de uno mismo o la de los demás, como resultado de la conducta individual. Esta conducta compromete el valor como persona, como consecuencia de su comportamiento, expresado como hacer algo que "no debe" o como no hacer algo que "debe hacer".
En la actualidad, se considera que la característica central o principal del pensamiento irracional es la rigidez, los "debo de" o "tengo que", los que fueron planteados inicialmente por Horney (1945) y de donde se derivan dichas inferencias o procesos irracionales secundarios. (Ellis, 1994; Lega, 1991)

La terapia racional emotivo-conductual, establece una diferencia muy importante entre la ansiedad del ego y la ansiedad situacional, en donde la ansiedad del ego se da como consecuencia de la condenación o "evaluación global de la valía del ser humano", mientras que la ansiedad situacional es producto de la baja tolerancia a la frustración (Ellis, 1980; 1987). La ansiedad del ego está estrechamente relacionada con la depresión mientras que la ansiedad situacional está relacionada con la intolerancia a situaciones de incomodidad y frustración.

La ansiedad del ego se define como la tensión emocional que resulta cuando las personas piensan, en primer lugar, que su valor como personas está siendo amenazado; en segundo lugar, que siempre "deben" comportarse perfectamente $\mathrm{y}$, en tercer lugar, que es horrible y catastrófico cuando fallan y/o si otras personas les rechazan cuando "deberían" aceptarlas. (Lega, Caballo y Ellis, 2002)

La ansiedad situacional, aunque inicialmente está relacionada con la poca tolerancia a situaciones de "incomodidad" y frustración o "peligro", puede generalizarse a sentimientos de ansiedad, depresión y vergüenza, asociados con dicha situación.

Ellis (1987) sostiene que la ansiedad situacional aparece más frecuentemente que la ansiedad del ego, pero al presentarse en una forma menos dramática, se identifica como un síntoma secundario, y muchas veces pasa desapercibida, o es mal diagnosticada, y se le llama "ansiedad generalizada".

En la década de 1960, Beck planteó la terapia cognitiva (TC), que tuvo gran difusión y reconocimiento por el enfoque 
que dio hacia las perturbaciones emocionales, sobre todo en lo concerniente a la depresión y a la ansiedad. En 1961 propuso un inventario auto-administrado para evaluar cuantitativamente la intensidad de las manifestaciones conductuales relacionadas con la depresión (DBI). (Beck et al., 1961)

Uno de los grandes aportes de este enfoque (Beck et al., 1979) fue el de identificar las distorsiones cognitivas, entre las que se pueden señalar: generalización excesiva, magnificación y minimización, personalización y pensamiento absolutista dicotómico, y se plantean como aquellos procesos cognitivos que cambian, por lo que podrían ser acontecimientos relativamente inofensivos, comparados con otros que se experimentan como adversos y que, como las ideas irracionales formuladas por Ellis, podrían conducir a emociones, conductas y consecuencias motivacionales negativas.

Beck y Emery (1985) plantean el hecho de que mientras en la depresión las actitudes y los esquemas cognitivos giran alrededor de situaciones de pérdida, en la ansiedad se hace alrededor de la amenaza. Ambas situaciones contemplan la pérdida y/o la amenaza como acontecimientos reales y potenciales.

Otro aspecto a tomar en consideración, en cuanto a la ansiedad versus depresión, es que en la primera el acontecimiento precipitante aún no se ha desencadenado en la persona, pero le preocupa en forma prioritaria, contrario a la depresión en donde el acontecimiento ya ha sucedido y la persona desarrolla sentimientos de culpabilidad.

Se han llevado a cabo comparaciones teóricas entre la TREC y la TC (Dryden y Ellis, 1988) y ambas indican que existe una relación entre inferencias y procesos secundarios (TREC) y distorsiones cognitivas (TC), y que a pesar de que el debate empírico se utiliza en ambos modelos, solo la TREC usa el debate filosófico para combatir la rigidez que se da en el pensamiento de los "debo de" actuar bien todo el tiempo, o si no se concluirá que "es terrible" que no lo haya hecho hasta ahora o los "tengo que" actuar bien en una situación determinada, pero como no lo he hecho hasta ahora, entonces no tengo la capacidad para hacerlo. Esta rigidez de pensamiento es el verdadero origen de la perturbación emocional.

Ellis $(1957 ; 1958 ; 1962)$ destaca que el centro de la ansiedad y la depresión es el pensamiento o filosofía grandiosa, los pensamientos rígidos y dogmáticos de la persona y no las inferencias o ideas secundarias, equivalentes a las distorsiones cognitivas, que Beck destaca como la causa principal.

El propósito de este estudio, es el de explorar, si realmente hay o no una relación empírica que se pueda establecer, entre los instrumentos Attitudes and Beliefs Inventory (ABI), (Escala de Actitudes y Creencias), mayormente utilizado en estudios empíricos sobre TREC, y el instrumento Beck Depression Inventory (Inventario de Depresión de Beck) en su segunda edición (BDI-II). Se seleccionaron ambos instrumentos para este estudio ya que han sido estandarizados para poblaciones de habla hispana.

\section{Metodología}

\section{Participantes}

Se seleccionó una muestra de 200 estudiantes de la Universidad de Costa Rica, de las Facultades de Ingeniería y Educación, y de las Escuelas de Química, Enfermería y Psicología. Los estudiantes participaron en este estudio en forma voluntaria.

\section{Instrumentos empleados}

Para la realización del trabajo experimental, se emplearon dos instrumentos diferentes.

En primer lugar, se utilizó el instrumento Attitudes and Beliefs Inventory o ABI (Burgess, 1990) que fue traducido y adaptado al español como "Escala de Actitudes y Creencias" (Caballo, Lega y González, 1986) y que es el que se ha utilizado en un mayor número de estudios transculturales (Lega 
y Ellis, 2001). Tomando en consideración que existen en América diferencias regionales en la utilización del idioma español, se prepararon varias versiones del test de actitudes $y$ creencias (ABT) (Lega y Ellis, 2001). Este instrumento está compuesto por siete sub escalas: tres niveles de contenido o áreas en las que se manifiesta la irracionalidad (aprobación, éxito y comodidad) y cuatro niveles de proceso (exigencia -tendencia primordial a pensar en forma rígida-, tremendismo, baja tolerancia a la frustración y condenación o "evaluación global de la valía del ser humano") que constituyen inferencias o procesos secundarios. Contiene 48 ítems, tipo Likert, los que se responden puntuando de 1 ("muy en desacuerdo") a 5 ("muy de acuerdo"). La versión española del ABT y la forma en que se calculan los puntajes correspondientes a los siete niveles mencionados se indicaron anteriormente. (Lega, Caballo, Ellis, 2002, págs. 41-47)

En segundo lugar, se utilizó el instrumento Beck Depression Inventory ("Inventario de Depresión de Beck") en su segunda edición (BDI-II y BAI-II: Beck, Steer y Brown, 1996). Este instrumento es el que mide mejor la depresión en adultos y adolescentes (Murphy, Impara y Plake, 1999) de acuerdo con la terapia cognitiva (TC). Este es un cuestionario que brinda un puntaje total, y también puntajes para los dos componentes de depresión (cognitivo-afectivo y somático). Es un inventario constituido por 21 ítems, utilizado ampliamente en investigación clínica.

\section{Procedimiento}

Se explicó a los participantes que el inventario presenta distintas actitudes y creencias que la gente mantiene a veces. Se leyeron en voz alta las instrucciones para llenarlo, lo que permitió asegurar que todos recibieran la misma información.
Esta información se encuentra en cada uno de los cuestionarios que se aplicaron.

Cada uno de los participantes respondió por escrito, en forma anónima e individual, a las preguntas, seleccionando un puntaje del 1 al 5 para indicar acuerdo o desacuerdo para cada uno de los 48 ítems del ABI (ABT), y un puntaje del cero al tres para los 21 ítems del BDI-II.

Para cada uno de los participantes fueron calculados los puntajes en las siete sub-escalas del ABI (aprobación, éxito, comodidad, exigencia, tremendismo, baja tolerancia a la frustración y condenación o evaluación global) identificadas por Burgess (1990) y los puntajes para los dos factores o componentes de depresión (cognitivo-afectivo y somático) del BDI-II, de cuerdo con Beck, Steer y Brown (1996).

\section{Análisis de los datos}

Para analizar los datos se utilizó el programa SPSS, versión 11.0, el que aplica para calcular coeficientes significativos de correlación (ro de Spearman) entre las siete sub-escalas del ABI y los dos componentes (cognitivo-afectivo y somático) del BDI-II.

\section{Resultados}

El programa SPSS, versión 13.0 produjo coeficientes significativos de correlación (ro de Spearman) entre las siete sub-escalas del ABI y el puntaje total del BDI-II $(\rho=0,05)$.

Como lo muestra la tabla 1, comparaciones post hoc (LSD) indicaron que, basado en el tamaño de los coeficientes, un gran componente de dichas correlaciones podría atribuirse a las correlaciones entre las subescalas de baja tolerancia a la frustración o BTF, comodidad y condenación o "evaluación global de la valía del ser humano", y el factor cognitivo-afectivo del BDI-II $(\rho=0,010)$. 
Tabla 1

Comparaciones entre las 7 sub-escalas del ABI y los dos componentes (cognitivo-afectivo y somático) del BDI-II en Costa Rica

\begin{tabular}{|c|c|c|}
\hline & BCK1COAF* & BCK2SOMA* \\
\hline \multicolumn{3}{|l|}{ EXIGENCIA } \\
\hline Coeficiente de correlación & 0,338 & 0,267 \\
\hline Nivel de significancia (1 dirección) & 0,01 & 0,01 \\
\hline \multicolumn{3}{|l|}{ TREMENDISMO } \\
\hline Coef. correlación & 0,323 & 0,260 \\
\hline Nivel de significancia (1 dirección) & 0,01 & 0,01 \\
\hline \multicolumn{3}{|l|}{ BTF } \\
\hline Coef. Correlación & 0,681 & 0,228 \\
\hline Nivel de significancia (1 dirección) & 0,01 & 0,01 \\
\hline \multicolumn{3}{|l|}{ CONDENACIÓN } \\
\hline Coef. correlación & 0,655 & 0,241 \\
\hline Nivel de significancia (1 dirección) & 0,01 & 0,01 \\
\hline \multicolumn{3}{|l|}{ APROBACIÓN } \\
\hline Coef. correlación & 0,391 & 0,213 \\
\hline Nivel de significancia (1 dirección) & 0,01 & 0,01 \\
\hline \multicolumn{3}{|l|}{ ÉXITO } \\
\hline Coef. correlación & 0,304 & 0,249 \\
\hline Nivel de significancia (1 dirección) & 0,01 & 0,01 \\
\hline \multicolumn{3}{|l|}{ COMODIDAD } \\
\hline Coef. Correlación & 0,644 & 0,635 \\
\hline Nivel de significancia (1 dirección) & 0,01 & 0,01 \\
\hline
\end{tabular}

*BCK1COAF = Beck Depression Inventory, factor 1, cognitivo-afectivo.

*BCK2SOMA = Beck Depression Inventory, factor 2, somático. 


\section{Discusión y conclusiones}

El resultado del análisis estadístico de los datos confirma empíricamente la relación entre ambos modelos en la siguiente forma:

(1) La terapia racional emotivo-conductual (Ellis) y la terapia cognitiva (Beck) tienen una base común por ser ambos modelos psicoterapéuticos representativos del enfoque cognitivo en psicoterapia, indicada por coeficientes significativos de correlación (ro de Spearman) entre las siete subescalas del ABI y el puntaje total de depresión del BDI-II $(\rho<0,05)$.

(2) La equivalencia teórica entre inferencias o procesos secundarios (TREC) y Distorsiones Cognitivas (TC) es corroborada por los resultados de las comparaciones post hoc (LSD), donde un gran componente de las correlaciones entre el ABI y el BDI-II puede ser atribuido a las correlaciones entre las sub-escalas de baja tolerancia a la frustración o BTF (ansiedad situacional), comodidad (ansiedad situacional) y condenación o "evaluación global de la valía del ser humano", (ansiedad del ego), y el factor cognitivo-afectivo del BDI-II ( $\rho=0,0001)$.

(3) Comparaciones post hoc (LSD) también corroboran la relación entre los factores somáticos en depresión (Beck) y la ansiedad situacional (Ellis), ya que el componente somático del BDIII se correlaciona únicamente con la sub-escala de comodidad (ansiedad situacional) del ABI $(\rho=0,01)$

(4) La correlación no significativa entre la sub-escala exigencia del ABI y el puntaje total de ansiedad del BAIII $(\rho>0,05)$ sugiere independencia entre la rigidez del pensamiento absolutista (los "debo de") y factores meramente somáticos en ansiedad, aunque en teoría podría darse una relación de causa-efecto entre ambas variables.
Matsumoto (2000) enfatiza la importancia de comparaciones transculturales para establecer la verdadera validez de las conclusiones científicas. Para ello, sugiere establecer equivalencia en el marco de referencia teórico y la metodología empírica en las investigaciones llevadas a cabo en varias culturas.

Los resultados de este estudio abren la posibilidad futura de explorar si existe o no esta relación empírica entre los modelos de Ellis (TREC) y de Beck (TC) en un contexto transcultural, utilizando los instrumentos existentes en sus versiones en español, en muestras de población hispano parlante.

\section{Referencias bibliográficas}

Beck, A. T. (1963). Thinking and Depression I. Idiosyncratic content and cognitive distortions, Archives of General Psychiatry, 9, 36-46.

Beck, A. T. (1976). Cognitive Therapy and Emotional Disorders. New York: International Universities.

Beck, A. T. y Emery, G. (1985). Anxiety disorders and phobias: A cognitive perspective. New York: Basic.

Beck, A. T., Rush, A. J., Shaw, B. F. y Emery, G. (1979). Cognitive Therapy of Depression. New York: Guilford.

Beck, A. T., Steer, R. A. y Brown, K. G. (1996). Manual for the Beck Depression Inventory. ( $2^{\mathrm{a}}$ ed.) San Antonio, TX: The Psychological Corporation.

Beck, A. T., Ward, C. H., Mendelson, M., Mock, J. y Erbaugh, J. (1961). An inventory for measuring depression. Arch. Gen. Psychiatry, 4, 561-571.

Burgess, P. M. (1990). Towards resolution of conceptual issues in the assessment of belief systems in Rational Emotive 
Therapy, Journal of Cognitive Psychotherapy: An International Quarterly, 4, 171-184.

Caballo, V. E., Lega, L. y González, S. (1986, noviembre). Factor analysis of a back-translated Spanish version of the scale of Attitudes and Beliefs. Ponencia presentada en el "30th Annual Convention of the Association for Advancement of Behavior Therapy", New York.

Dobson, K. S. (1986). The self-schema in depression. In L. M. Hartman y K. R. Blankestein (Comp.), Perception of self and emotional disorders in psychotherapy. New York: Plenum.

Dryden, W. y Ellis, A. (1988). RationalEmotive Therapy. In K. S. Dobson (Dir.), Handbook of cognitive-behavioral therapies. New York: Guilford.

Ellis, A. (1955). Psychotherapy techniques for use with psychotics. American Journal of Psychotherapy 9, 452-476.

Ellis, A. (1957). Outcome of employing three techniques of psychotherapy. Journal of Clinical Psychology 13, 344-350.

Ellis, A. (1958). Rational psychotherapy. Journal of General Psychology, 59, 35-49.

Ellis, A. (1962). Reason and emotion in psychotherapy. New York: Lyle Stuart.

Ellis, A. (1973). Humanistic psychotherapy: The RET approach. New York: Mc-Graw Hill.

Ellis, A. (1975). How to live with a neurotic. North Hollywood, CA: Wilkshire.

Ellis, A. (1976). RET abolishes most of the human ego. Psychotherapy: Theory, research \& practice, 13, 343-348.
Ellis, A. (1980). Discomfort anxiety: A new cognitive-behavioral construct. Rational Living, 15(1), 25-30.

Ellis, A. (1984 b). The essence of RET. Journal of Rational Emotive Therapy, 2(1), 19-25.

Ellis, A. (1985). Overcoming resistance: RET with difficult clients. New York: Springer.

Ellis, A. (1987). A sadly neglected element in depression, Cognitive Therapy and Research, 11, 121-146.

Ellis, A. y Abrahms, E. (2001). Terapia racional-emotiva (TRE). Mejor salud y superación personal afrontando nuestra realidad. México, D.F.: Editorial Pax México.

Ellis, A. y Becker, I. (1982). A guide to personal happiness. North Hollywood. Ca: Wilkshire.

Ellis, A. y Bernad, M. (1990). ¿Qué es la terapia racional emotiva (RET)? En A. Ellis y M. Bernard (Dirs.), Aplicaciones clínicas de la terapia racional-emotiva (pp. 19-48) Bilbao: Desclée de Browerr.

Ellis, A. y Bernad, M. (1994). Reason and Emotion in Psychotherapy: Revised and updated. New York: Birch Lane.

Epitectus (1980). The colected Works of Eptectus. Boston: Little Brown.

Grieger, R. y Boyd, J. (1980). Rational Emotive Therapy: A skills based approach. New York: Van Nostrand Reinhold.

Horney, K. (1945). Our Inner Conflicts: A constructive theory of neurosis. New York: Norton. 
Lega, L. (1991). La Terapia Racional Emotiva: Una conversación con Albert Ellis. In V. E. Caballo (Dir.), Manual de Técnicas de Terapia y Modificación de Conducta. Madrid: Siglo XXI.

Lega, L. y Ellis, A. (2001). REBT in the New Millenium: A cross-cultural approach, Journal of Rational-emotive and Cognitive-behavioral Therapies, 19(4), 201-222.

Lega, L., Caballo, V. E. y Ellis, A. (2002). Teoría y práctica de la terapia racional emotivo-conductual. ( $2^{\text {a }}$ ed.). Madrid: Siglo XXI.
Matsumoto, D. R. (2000). Culture \& Psychology: People around the World. $\left(2^{\text {a }}\right.$ ed.). Stamford, CT: Wadsworth.

Murphy, L. L., Impara, J. C., y Plake, B. S. (1999). Tests in Print V. Lincoln, Nebraska: Buros.

Sutton-Simon, K. (1981). Assessing belief systems: Conceptions and strategies. In P. C. Kendall and S. D. Hollon (Dir.), Assessment Strategies for Cognitive Behavioral Interventions. New York: Academic. 